\title{
(6) OPEN ACCESS \\ Effects of flashlight guidance on chest compression performance in cardiopulmonary resuscitation in a noisy environment
}

\author{
Je Sung You, ${ }^{1}$ Sung Phil Chung, ${ }^{1}$ Chul Ho Chang, ${ }^{2}$ Incheol Park, \\ Hye Sun Lee, ${ }^{3}$ SeungHo Kim, ${ }^{1}$ Hahn Shick Lee ${ }^{1}$
}

${ }^{1}$ Department of Emergency Medicine, Yonsei University College of Medicine, Seoul, Republic of Korea

${ }^{2}$ Department of Anesthesiology and Pain Medicine, Yonsei University College of Medicine, Seoul, Republic of Korea ${ }^{3}$ Department of Biostatistics, Yonsei University College of Medicine, Seoul, Republic of Korea

\section{Correspondence to} Incheol Park, Department of Emergency Medicine, Yonsei University College of Medicine, 50 Yonsei-Ro, Seodaemun-Gu, Seoul 120-752, Republic of Korea; incheol@yuhs.ac

Accepted 11 July 2012

Published Online First 27 August 2012

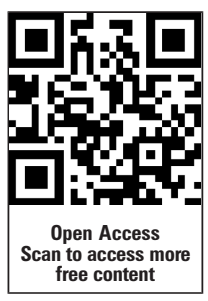

\begin{abstract}
Background In real cardiopulmonary resuscitation (CPR), noise can arise from instructional voices and environmental sounds in places such as a battlefield and industrial and high-traffic areas. A feedback device using a flashing light was designed to overcome noise-induced stimulus saturation during CPR. This study was conducted to determine whether 'flashlight' guidance influences CPR performance in a simulated noisy setting. Materials and methods We recruited 30 senior medical students with no previous experience of using flashlight-guided CPR to participate in this prospective, simulation-based, crossover study. The experiment was conducted in a simulated noisy situation using a cardiac arrest model without ventilation. Noise such as patrol car and fire engine sirens was artificially generated. The flashlight guidance device emitted light pulses at the rate of 100 flashes/min. Participants also received instructions to achieve the desired rate of 100 compressions/min. CPR performances were recorded with a Resusci Anne mannequin with a computer skillreporting system.
\end{abstract}

Results There were significant differences between the control and flashlight groups in mean compression rate (MCR), MCR/min and visual analogue scale. However, there were no significant differences in correct compression depth, mean compression depth, correct hand position, and correctly released compression. The flashlight group constantly maintained the pace at the desired 100 compressions/min. Furthermore, the flashlight group had a tendency to keep the MCR constant, whereas the control group had a tendency to decrease it after $60 \mathrm{~s}$.

Conclusion Flashlight-guided CPR is particularly advantageous for maintaining a desired MCR during hands-only CPR in noisy environments, where metronome pacing might not be clearly heard.

\section{INTRODUCTION}

The 2010 American Heart Association (AHA) and European Resuscitation Council (ERC) cardiopulmonary resuscitation (CPR) guidelines have been optimised for high-quality CPR. Chest compression quality is of the utmost importance for a patient's survival after cardiac arrest. ${ }^{12}$

Many studies have reported that audiovisual feedback systems improve CPR quality. Metronome guidance is the simplest and cheapest feedback system. ${ }^{3}$ Audio-prompt rate guidance significantly improves chest compression rate and mean end-tidal $\mathrm{CO}_{2}$ concentration during $\mathrm{CPR}^{4-6}$
Oh et al found that metronome use increased the mean compression rate (MCR) to the desired value, but compression depth decreased because of the requirement to multitask (CPR technique and audio perception of audio tones). ${ }^{7}$

Stimulus saturation limits signal recognition during complex situations such as resuscitation. This effect can lead to frustration, reduced performance and increased human error. ${ }^{8}$ In real CPR situations, noise can arise from instructional voices and environmental sounds in places such as battlefields and industrial, commercial shopping and high-traffic areas. WHO suggested guideline limits for community noise in specific environments. In the environments mentioned above, noise levels were measured to be $>70 \mathrm{~dB}$, which can induce irritation and hearing impairment. ${ }^{9}$ A simple feedback device using a flashing light stimulus was designed to overcome noise-induced stimulus saturation during CPR. This study was conducted to determine whether flashlight guidance influences CPR performance in a simulated noisy setting.

\section{MATERIALS AND METHODS Participants}

We recruited 30 senior medical students with no previous experience of using flashlight-guided CPR to participate in this prospective, simulation-based, crossover study. The study was approved by the institutional review board. Written informed consent was obtained from all subjects. All participants had previous CPR training in which adult basic life support was taught according to 2010 AHA guidelines, ${ }^{1}$ using a training mannequin. This training lasted $6 \mathrm{~h}(2 \mathrm{~h}$ of lecture and $4 \mathrm{~h}$ of practical training), in accordance with 2010 AHA guidelines for CPR to be taught as part of a regular college course. The training was given at least 6 months before this study.

\section{Study protocol}

All participants were given 20 min of instruction concerning flashlight guidance CPR and an overview of this study. The memory rate of 100 ticks/ min was provided by a metronome. In a simulated noisy situation, the experiment was conducted using a cardiac arrest model with hands-only CPR. Noise such as patrol car and fire engine sirens was artificially generated (Sirens-iPhone application; BrennanMoyMedia, New York, New York, USA). Siren noise intensity was adjusted to $80 \pm 5 \mathrm{~dB}$, 
with 60 pitches/min, by a sound dose meter (DT-805L; Gunpoong Electronics, Busan, Korea). We designed and manufactured the flashlight guidance device (Top-Lighting, Seoul, Korea). It emitted light pulses at the rate of 100 flashes/min from a light-emitting diode. When participants performed CPR with flashlight guidance, they received instructions equivalent to the rate of the flashlight guidance. When participants performed CPR without flashlight guidance, they also received instructions suited to achieving the desired rate of 100 compressions/min. CPR was randomly performed in one of two sequences. In sequence $\mathrm{A}$, the participant conducted CPR with flashlight guidance. After a $30 \mathrm{~min}$ rest period, the participant conducted CPR without flashlight guidance. In sequence $\mathrm{B}$, the participant conducted CPR without flashlight guidance. After a 30 min rest period, the participant conducted CPR with flashlight guidance. Single-rescuer adult basic life support was performed on the mannequin for $2 \mathrm{~min}$ by all participants. The sequence selection for CPR performance was computer randomised. Skill assessment was performed according to the 2010 AHA guidelines. A Resusci Anne mannequin with a personal computer skill-reporting system (Laerdal, Stavanger, Norway) was used to assess CPR performance.

\section{Outcome assessment}

Compression rate and depth and percentage of compressions with correct hand positioning were recorded with a Resusci Anne mannequin with a personal computer skill-reporting system. Participants were asked to report on 'rescuer fatigue' on a visual analogue scale of $0-10$ ( 0 , extremely easy; 10 , extremely difficult). Open questions were posed to all participants on the strengths and weaknesses of performing chest compressions with or without flashlight guidance in a noisy situation. After answers to the open questions had been collected, all participants were questioned again to achieve consistency.

\section{Statistical analysis}

Sample size was calculated according to our primary end points. Differences of five compressions/min and $5 \mathrm{~mm}$ in mean compression depth between the two methods were selected as minimum clinically significant values. Using these values, we calculated that a sample size of 28 participants was sufficient to detect an effect value of 0.75 (mean difference/common SD) at a significance level of 0.05 (two-sided) with $80 \%$ power. A linear mixed model for $2 \times 2$ crossover design was used. Three fixed effects were included: one between-subject effect, which was the sequence effect (CPR without flashlight guidance for the first half of the subjects and then CPR with flashlight guidance for the second half; CPR with flashlight guidance for the first half of the subjects and then CPR without flashlight guidance for the second half); two within-subject effects, which was the group effect (CPR with flashlight guidance, CPR without flashlight guidance); the period effect (first, second). Subject was considered as random effect. Since a half of the subjects were assigned to one sequence and the rest were assigned to the other half, they were nested within sequence.

A linear mixed model for evaluating changes in chest compression rate across time was also used. Two fixed effects were included: one between-subjects effect, which was the group effect (CPR with flashlight guidance, CPR without flashlight guidance); and one within-subject effect, which was the time effect $(30,60,90,120 \mathrm{~s})$. A possible difference in sequence across $30-120 \mathrm{~s}$ was analysed by group $\times$ time interaction. The group $\times$ time interaction was tested at a significance level of 0.05. Multiple comparisons with the Bonferroni correction as the post hoc analysis were used to compare the point of rate change that was significantly different from the baseline. All statistical analyses were conducted using SAS V.9.2.

\section{RESULTS}

\section{Variables of CPR performance}

All 30 participants completed the study. The median participant age was 25 (IOR 25-26) years, and 22 (73.3\%) participants were male. Results from the linear mixed model are displayed in table 1, including outcome and group differences from baseline to 120 s. p Values of main effects for sequence, period and group are given. The sequence did not affect the results for all performance variables, and there were no significant differences between the first and second periods. There were significant differences between the control and flashlight guidance groups for $\mathrm{MCR}, \mathrm{MCR} / \mathrm{min}$ and visual analogue scale. However, there were no significant differences for correct compression depth $(\%)$, mean compression depth ( $\mathrm{mm})$, correct hand position (\%) and correctly released compression (\%).

\section{MCR at the desired rate of $100 / \mathrm{min}$}

Results of MCR at the desired rate (100 compressions/min) are shown in figure 1 . The MCR of the flashlight guidance group was constantly maintained at the desired 100 compressions $/ \mathrm{min}$ (estimated mean 100.8 compressions/min (95\% CI 97.5 to 104.1)), whereas that of the control group was distributed over a much wider range (estimated mean 120.7 (95\% CI 117.4 to 124.1)).

\section{Changes in chest compression across time}

Effects obtained for time $(p=0.01)$ by group $(p<0.01)$ and interactions $(p=0.01)$ suggest that changes from 0 to $120 \mathrm{~s}$ were in general different between the groups. Post hoc analyses were conducted for the point of rate changes. No differences in MCR

Table 1 Results from a linear mixed model for cardiopulmonary resuscitation performance

\begin{tabular}{|c|c|c|c|c|c|c|}
\hline \multirow[b]{2}{*}{ Variable } & \multirow[b]{2}{*}{ Control } & \multirow[b]{2}{*}{ Flashlight } & \multirow[b]{2}{*}{ Difference (control-flashlight) } & \multicolumn{3}{|l|}{ p Value } \\
\hline & & & & Sequence & Period & Group \\
\hline Mean compression depth (mm) & 54.7 (52.9 to 56.5$)$ & 54.7 (52.9 to 56.5$)$ & $<-0.001(-0.92$ to 0.92$)$ & 0.43 & 0.77 & $>0.99$ \\
\hline Correct compression depth $(\%)^{*}$ & 82.1 (71.9 to 92.3$)$ & 84.7 (74.5 to 94.97$)$ & $-2.63(-8.05$ to 2.78$)$ & 0.39 & 0.54 & 0.33 \\
\hline Mean compression rate & $120.4(117.0$ to 123.7$)$ & 100.7 (97.4 to 104.1$)$ & 19.63 (14.98 to 24.28$)$ & 0.35 & 0.33 & $<0.01$ \\
\hline Mean compression rate/min & 120.7 (117.4 to 124.1$)$ & 100.8 (97.5 to 104.2$)$ & $19.90(15.30$ to 24.50$)$ & 0.35 & 0.31 & $<0.01$ \\
\hline Correct hand position (\%) & 87.6 (80.7 to 94.5$)$ & $84.2(77.3$ to 91.1$)$ & $3.40(-0.23$ to 7.03$)$ & 0.50 & 0.41 & 0.06 \\
\hline Correctly released compression (\%) & 97.5 (93.8 to 101.2 ) & $96.6(92.9$ to 100.3$)$ & $0.97(-1.57$ to 3.51$)$ & 0.52 & 0.30 & 0.44 \\
\hline Rescuer fatigue (VAS) & $6.2(5.6$ to 6.9$)$ & $4.8(4.2$ to 5.4$)$ & $1.42(0.52$ to 2.31$)$ & 0.95 & 0.51 & $<0.01$ \\
\hline
\end{tabular}

Values are estimated mean $(95 \% \mathrm{Cl})$.

*Correct compression depth is $>50 \mathrm{~mm}$

VAS, visual analogue scale of $0-10(0$, extremely easy; 10 , extremely difficult). 
Figure 1 Maintenance of mean compression rate at a desired rate $(100$ compressions/min).

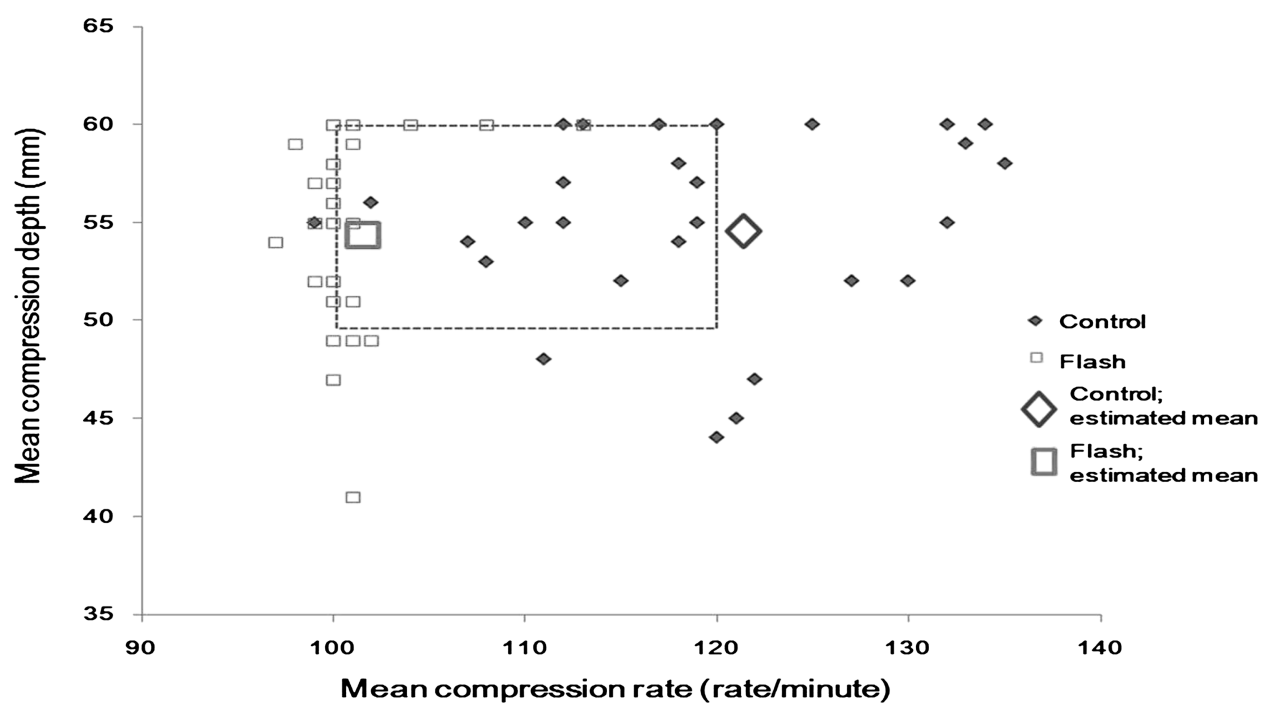

were seen in time $\times$ group interactions from 30 to $60 \mathrm{~s}$ ( $\mathrm{p}>0.99)$. However, effects were observed for time $\times$ group interactions from 30 to $90 \mathrm{~s}(\mathrm{p}=0.04)$ and from 30 to $120 \mathrm{~s}(\mathrm{p}=0.03)$. The results suggest that the flashlight guidance group had a tendency to maintain constant MCR, whereas the control group had a tendency to decrease MCR after $60 \mathrm{~s}$ (figure 2).

\section{Open questionnaire}

In open questions, 28 of 30 participants (93.3\%) responded that flashlight guidance made it easier to adjust CPR to the desired rate. The majority of participants (24 of $30 ; 80.0 \%$ ) reported that it was difficult to mentally count compressions to maintain the desired MCR because they were affected by the noisy sirens. Twelve of the participants (40.0\%) responded that the flashlight guidance during CPR distracted them from verifying the correct hand position. More than half of the participants (16 of 30; $53.3 \%$ ) responded that the noise of a slow siren (60 pitches/min) caused an excessive increase in MCR.

\section{DISCUSSION}

Our study demonstrates that flashlight guidance influences maintenance of the desired MCR during CPR and reduces rescuer fatigue, similar to the effects of a metronome. The 2010 AHA guidelines recommend an MCR of at least 100 compressions/min and a depth of at least $5 \mathrm{~cm}$. According to 2005 AHA/ERC guidelines, many researchers considered that a compression rate of about 100/min could not be reached. Recent 2010 AHA/ERC guidelines emphasised more strongly than previous guidelines the concept of 'push hard, push fast'. Compared with the flashlightguided group, the MCR of the control group was significantly increased above the desired rate in noisy situations. Field et al ${ }^{10}$ found that a chest compression rate above $120 /$ min reduces compression quality. They and the 2010 ERC guidelines recommended that a MCR of 100-120 compressions/min for $2 \mathrm{~min}$ is feasible for maintaining adequate chest compression quality. ${ }^{2}{ }^{10} \mathrm{It}$ has been shown that metronome-guided rate-directed CPR increases end-tidal $\mathrm{CO}_{2}$ concentration and improves survival rates. ${ }^{4} 11$ Metronome guidance has been shown to correct compression rates to within the guidelines. ${ }^{11}$ Flashlight guidance $\mathrm{CPR}$, like metronome guidance, can maintain the pace by correcting excessive decreases and increases in compression rates. Maintenance of pace of chest compression using flashlight guidance can be beneficial in decreasing rescuer fatigue and correcting compression rates that have fallen in noisy environments.
Figure 2 Changes in mean compression rate (MCR) across time. The results suggest that the flashlight guidance group had a tendency to maintain MCR, whereas the control group had a tendency to decrease MCR after $60 \mathrm{~s}$ (indicated by the star).

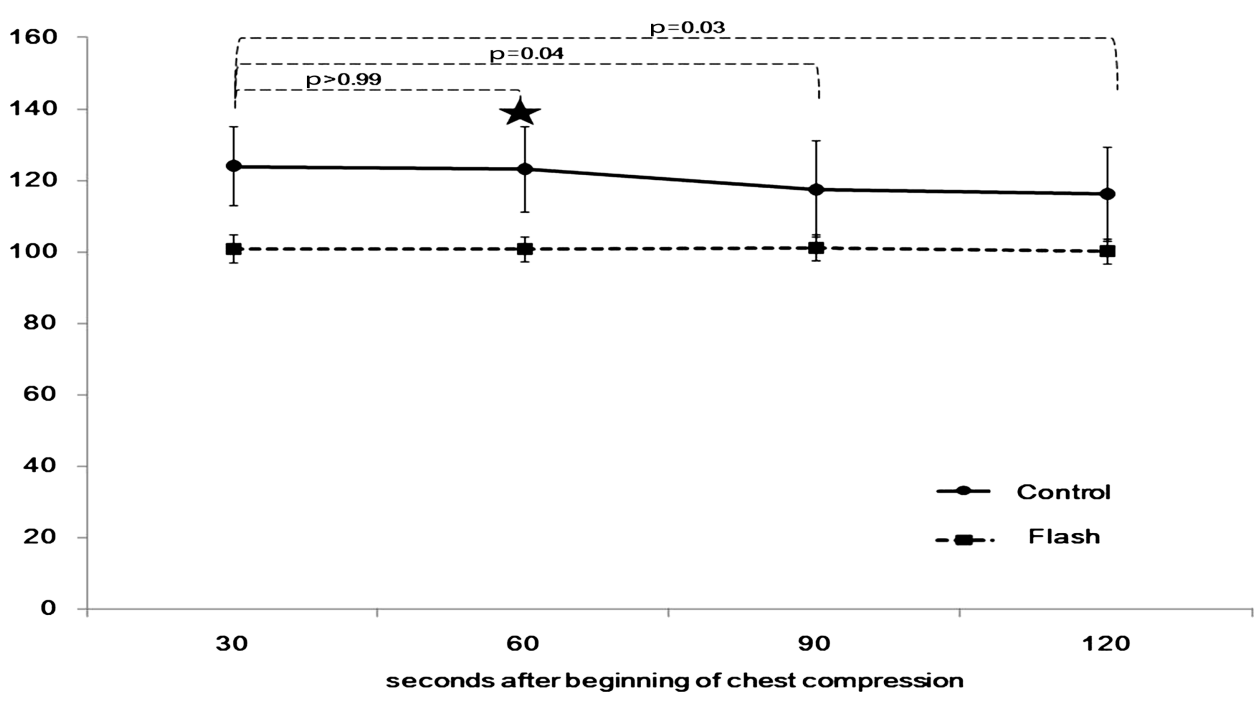


There were no significant differences between the two groups with respect to compression depth and appropriate compression depth (\%). The compression depth results can be viewed as education effects resulting from the 'push hard' CPR training emphasised by the 2010 AHA/ERC guidelines. ${ }^{1} 2$

For improving CPR quality, maintenance of the appropriate compression rate and depth is a primary consideration. Although further study is needed, CPR performance has been found to be decreased by noise such as sirens, instructions, voices and other environmental sounds. Emergency patients requiring $\mathrm{CPR}$ can be encountered anytime and anywhere. Emergency situations often occur in noisy places such as battlefields, festivals, industrial and commercial districts, shopping centres, and high-traffic areas. CPR feedback systems used in prehospital settings should be simple and low cost. Until recently, audio-tone guidance has been considered to be the standard feedback system for public CPR. However, audioguidance may not be effective in noisy situations. Light signals are a potential alternative to or supplementation for audioguided CPR. Light travels much faster than sound and has additional obvious benefits when CPR must be performed in a dark place. The flashing light can facilitate rapid recognition of CPR guidance at greater distances. Abella et al reported that resuscitation complexity increases the possibility of human error, leading to decreased performance. ${ }^{12}$ The stimulus saturation effect limits signal recognition during complex situations such as resuscitation. This effect can lead to frustration and reduced CPR performance. ${ }^{8} 13$ Light is a new and effective alternative pacing stimulus because CPR itself creates extraneous auditory stimuli. Although our study found that flashlight guidance distracted $40 \%$ of participants from hand position correction, new light stimuli development and CPR training regimens should be able to overcome this.

Additional training courses are strongly encouraged to achieve adequate CPR performance. A variety of flashlight devices for CPR could be produced and distributed to the general public to encourage nationwide use of flashlight-guided CPR, thus generally improving resuscitation effectiveness. These flashlights could be attached to key rings, necklaces or mobile phones. If the flashlight rate can be standardised with the desired MCR, in an emergency situation, calibrated directional indicators in cars and light bars in emergency vehicles could be used as flashlightguidance CPR devices (figure 3).

Photosensitivity is abnormal sensitivity to light stimuli, and in humans is usually in the frequency range $10-30 \mathrm{~Hz} .{ }^{14}$ Most patients are sensitive to stimulation of $16 \mathrm{~Hz}$. Almost half of the population (49\%) is sensitive to $50 \mathrm{~Hz}$, and only $15 \%$ is sensitive to $60 \mathrm{~Hz}$, which are the television frequencies in Europe and North America, respectively. ${ }^{13}$ About 3\% of the photosensitive population with some degenerative disorder is sensitive to intermittent photic stimulation at 1-3 flashes/second. ${ }^{15}$ The flashlight rate for CPR guidance is usually between 1.7 and 2 flashes/s (1.7-2 Hz). Thus such flashlights could be safely used to improve CPR performance.

\section{Study limitations}

There are several limitations to our study. First, we used mannequins as models to simulate an emergency resuscitation situation. This simulation could not reproduce the real nature of emergency situations. Second, laypeople can be confused by additional visual CPR-guidance stimuli in noisy environments. Flashlights that are too bright or of certain colours may have a disturbing effect. Application of flashlight guidance may increase the complexity of $\mathrm{CPR}$, thus delaying its initiation by

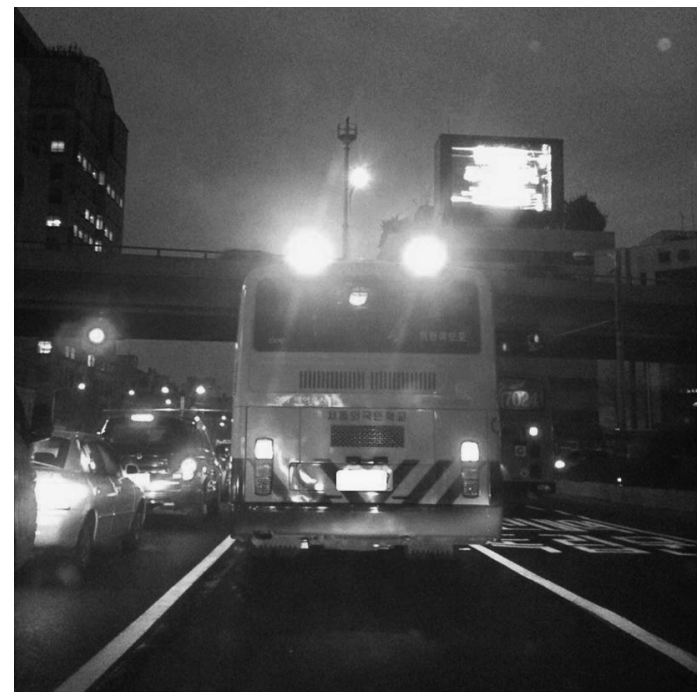

Figure 3 A school bus warning light could function as a flashlightguidance cardiopulmonary resuscitation device.

inexperienced laypeople. However, this study suggests that this problem can be overcome by training and nationwide campaigns. Further studies should be considered to investigate flashlights for comfortable CPR performance. Third, the participants may have had atypical motivations for and emotional responses to participation in this study. These participants were not laypeople, but medical students trained according to the 2010 AHA guidelines. We observed no significant differences between performance variables among the two test groups except for maintenance of the desired MCR. It is likely that a lay rescuer would perform lower-quality CPR. Compression depth by inexperienced lay rescuers may be one difference between the two groups. Fourth, our study showed that subjective fatigue of participants using flashlight-guided CPR was less than that in the control group in noisy environments. However, this may not be the result of the flashlight itself, but the result of preventing excessive MCR during CPR performance in a noisy environment.

At present, the impact of the flashlight-guided CPR on patient outcome has yet to be evaluated in actual noisy emergency situations. Our study demonstrated that both flashlight- and metronome-based CPR guides are simple and economic feedback systems, with a similar beneficial effect on CPR performance. Further studies are required to determine the effect of flashlightguided CPR performance when adjusting the MCR to between 110 and 120 compressions/min, according to the 2010 AHA/ ERC guidelines, and on the clinical results of flashlight-guided CPR performed by laypeople in prehospital settings.

\section{CONCLUSION}

Flashlight guidance is an effective low-cost CPR feedback system for maintaining the appropriate compression rate. It may be particularly advantageous during hands-only CPR in noisy environments where audio-tone cannot be clearly heard.

Contributors Guarantors of integrity of the entire study: JSY, SPC, IP. Study concepts and design: JSY, SPC, IP, CHC, HySL, SHK. Literature research: JSY, SPC, IP, CHC, SHK, HaSL. Clinical studies: JSY, SPC, IP. Experimental studies/data analysis: JSY, SPC, IP, CHC. Statistical analysis: JSY, SPC, IP, HySL. Manuscript preparation: JSY, SPC, IP, CHC. Manuscript editing: JSY, SPC, IP, SHK, HaSL, CHC

Funding This study was supported by a faculty research grant to Yonsei University College of Medicine for 2011(6-2011-0124). 


\section{Competing interests None.}

Ethics approval Ethics approval was provided by Yonsei University College of Medicine, Severance Hospital.

Provenance and peer review Not commissioned; externally peer reviewed.

Open Access This is an Open Access article distributed in accordance with the Creative Commons Attribution Non Commercial (CC BY-NC 3.0) license, which permits others to distribute, remix, adapt, build upon this work non-commercially, and license their derivative works on different terms, provided the original work is properly cited and the use is non-commercial. See: http://creativecommons.org/licenses/by-nc/3.0/

\section{REFERENCES}

1. Travers AH, Rea TD, Bobrow BJ, et al. Part 4: CPR overview: 2010 American Heart Association Guidelines for cardiopulmonary resuscitation and emergency cardiovascular care. Circulation 2010;122(18 Suppl 3):S676-84.

2. Koster RW, Baubin MA, Bossaert LL, et al. European Resuscitation Council Guidelines for Resuscitation 2010 Section 2. Adult basic life support and use of automated external defibrillators. Resuscitation 2010;81:1277-92.

3. Chung TN, Kim SW, You JS, et al. The specific effect of metronome guidance on the quality of one-person cardiopulmonary resuscitation and rescuer fatigue. J Emerg Med. Published Online First: 23 March 2012. doi:10.1016/j.jemermed.2012.01.021

4. Kern KB, Sanders AB, Raife J, et al. A study of chest compression rates during cardiopulmonary resuscitation in humans. The importance of rate-directed chest compressions. Arch Intern Med 1992;152:145-9.
5. Milander MM, Hiscok PS, Sanders AB, et al. Chest compression and ventilation rates during cardiopulmonary resuscitation: the effects of audible tone guidance. Acad Emerg Med 1995;2:708-13.

6. Berg RA, Sanders AB, Milander M, et al. Efficacy of audio-prompted rate guidance in improving resuscitator performance of cardiopulmonary resuscitation on children. Acad Emerg Med 1994;1:35-40.

7. $\mathbf{O h} \mathbf{~ J H}$, Lee SJ, Kim SE, et al. Effects of audio tone guidance on performance of CPR in simulated cardiac arrest with an advanced airway. Resuscitation 2008;79:273-7.

8. Bohn A, Weber TP. Wecker $S$, et al. The addition of voice prompts to audiovisual feedback and debriefing does not modify CPR quality or outcomes in out of hospital cardiac arrest-a prospective, randomized trial. Resuscitation 2011:82:257-62.

9. Berglund B, Lindvall T, Schwela DH. Guidelines for Community Noise. World Health Organization, 1999:55-65

10. Field RA, Soar J, Davies RP, et al. The impact of chest compression rates on quality of chest compressions-a manikin study. Resuscitation 2012;83:360-4.

11. Kern KB, Stickney RE, Gallison L, et al. Metronome improves compression and ventilation rates during CPR on a manikin in a randomized control. Resuscitation 2010;81:206-10

12. Abella BS, Kim S, Edelson DP, et al. Difficulty of cardiac arrest rhythm identification does not correlate with length of chest compression pause before defibrillation. Crit Care Med 2006;34(12 Suppl):S427-31.

13. Rittenberger JC, Guimond G, Platt TE, et al. Quality of BLS decreases with increasing resuscitation complexity. Resuscitation 2006;68:365-9.

14. Verrotti A, Tocco AM, Salladini C, et al. Human photosensitivity: from pathophysiology to treatment. Eur J Neurol 2005;12:828-41.

15. Zifkin BG, Kasteleijn-Nolst Trenité D. Reflex epilepsy and reflex seizures of the visual system: a clinical review. Epileptic Disord 2000;2:129-36. 